\title{
Swoop and swerve
}

\section{Spinning Flight: Dynamics of Frisbees, Boomerangs, Samaras and Skipping Stones \\ by Ralph D. Lorenz \\ Springer: 2006.346 pp. $€ 24, \$ 49.95$}

\section{H. K. Moffatt}

What do cricket balls, frisbees, boomerangs, rotorcraft, samaras (the winged fruits of trees such as the ash or maple) and 'sensor fused weapons' all have in common? Answer: they spin as they swoop as they swerve, and are subject to a combination of aerodynamic and gyroscopic effects that determine their translational and rotational motion. In his fascinating book Spinning Flight, Ralph Lorenz provides a rich feast of examples of such spinning bodies, some occurring naturally, some contrived for pleasure or for a practical purpose, some exotic. He discusses the flight of each within the common framework of Euler's principles of rigid-body dynamics together with the principles of aerodynamics insofar as these determine the net force and torque experienced by the body.

Lorenz is a space scientist who worked on the control of the Huygens probe, which in early 2005 made its gyroscopic descent through the atmosphere of Saturn's moon Titan. This experience, involving the design of sophisticated on-board instrumentation, led to his wider interest in spinning flight - initially that of the ever-popular frisbee - and to recognition of the insights that might be gained from attaching miniaturized sensors to record the details of flight path and spin.

Whether in sport or in casual recreation, the side force associated with spin is a familiar phenomenon: for golfers prone to slice, it is usually a source of frustration, whereas in cricket it is exploited by bowlers to achieve deceptive swerve. This side force arises because the spin, or 'vorticity', imparted to the air in the immediate vicinity of the ball is swept into a downstream wake in the form of a pair of vortices, like those shed from the tips of aircraft wings. If backspin, say, is imparted to the ball, these vortices propagate downwards, carrying momentum with them; the associated reaction of the air on the ball is an upward force perpendicular both to the motion and the axis of spin (the Robins-Magnus effect). Although fully understood for cylindrical bodies, a rigorous derivation of this transverse force for spheres or more complex bodies is lacking, clear though the physical mechanism maybe.

Children playing table-tennis soon learn how to impart topspin, backspin or sidespin to the ball, and it is here that the effect of the transverse force is most evident. In cricket, the rough seam of the ball is a deliberate complication; it triggers the transition to turbulence in the boundary layer, delaying flow separa-

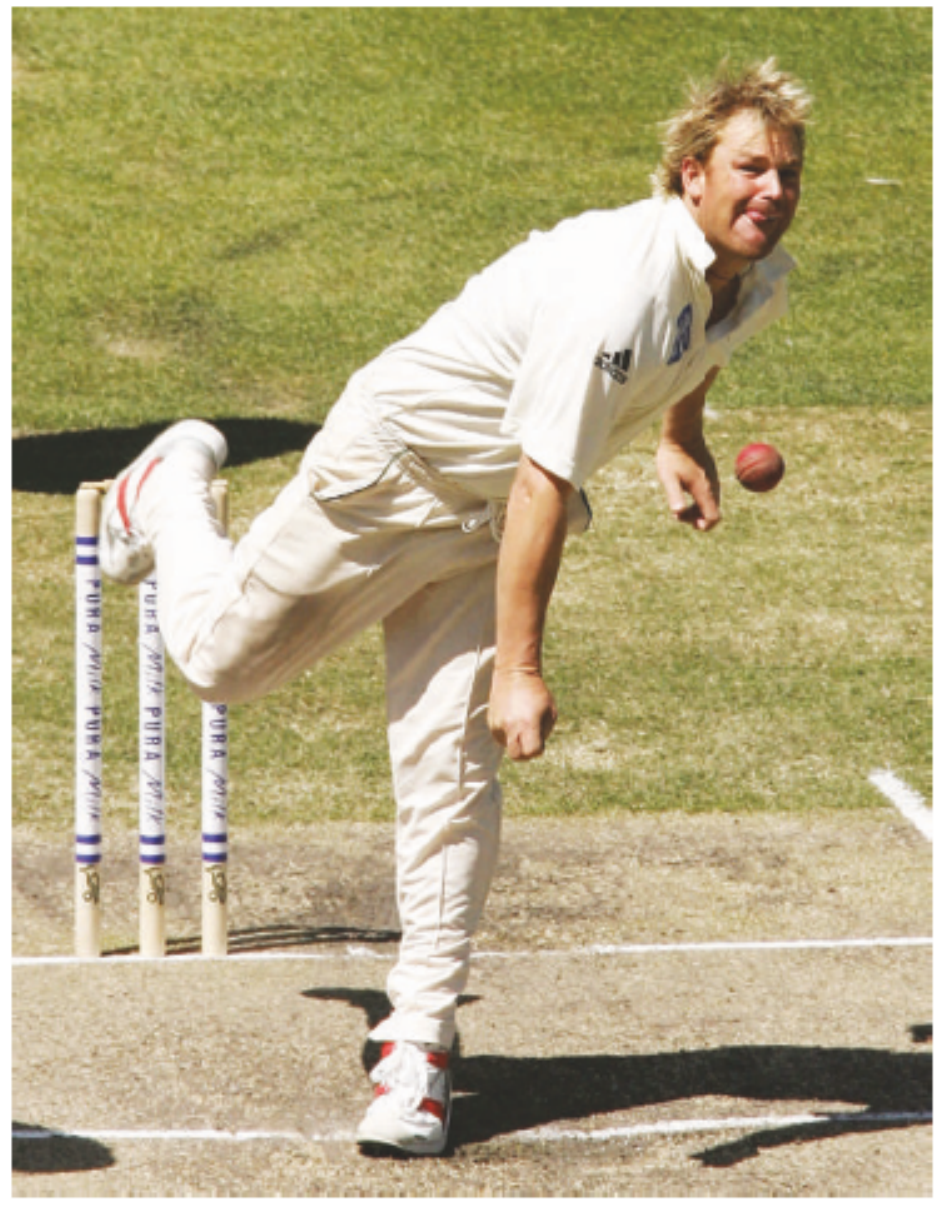

Wrist action: the spin imparted on the cricket ball by bowler Shane Warne has helped Australia to lead the current Ashes series against England.

tion and leading, paradoxically, to a decrease in drag. For a spinning ball, the orientation of the seam is critical. The dimples on a golf ball reduce drag for the same reason, and so increase its range if well struck. Again, in terms of fluid dynamics, the physical effect is well understood, but unresolved problems concerning the optimal scale and shape of the dimples continue to challenge, for reasons both aesthetic and commercial. The degree to which a golfclub can impart spin to the ball is a matter of impact dynamics, for which both the roughness and the compliance of the impacting surfaces are essential ingredients.

For non-spherical bodies such as a frisbee or boomerang, questions of gyroscopic stability are central. If external torques and internal dissipation (due, for example, to flexural deformation) are negligible, then, as Euler knew more than 250 years ago, such a body can spin stably about its axis of either greatest or least moment of inertia, whereas spin about the axis of intermediate inertia is unstable. The presence of aerodynamic torques modifies these conclusions, but they nevertheless provide a good starting point for a general dynamical treatment.

Lorenz covers these phenomena and their various manifestations with great skill and economy of exposition, whetting the appetite for the more detailed treatments that he cites in an extensive list of references, many quite recent. The book is well organized, progressing from simple to more complex systems, culminating in a discussion of skipping stones (I was astonished to learn that the current record is 40 skips) and the related 'bouncing bomb' developed by Barnes Wallis and used to such effect during the Second World War - just one example of the way recreational pursuits can inspire technological development.

The discussion in the book is deliberately non-technical, and should be accessible to readers with some elementary understanding of aerodynamic principles. For the expert, the book is full of open problems that cry out for detailed investigation, whether analytical, experimental or computational. Its scope is extensive, ranging from samaras at one extreme to aircraft such as the EC-2 Hawkeye, with its rotating radar antenna, or radome, at the other. In this respect, there may be something for everyone within its attractively designed cover: a good Christmas present for the man who has everything.

H. K. Moffatt is in the Department of Applied

Mathematics and Theoretical Physics, Wilberforce Road, Cambridge CB3 OWA, UK 